MANCHESTER

1824

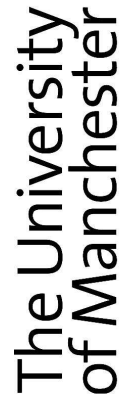

A Hyperbolic Augmented Elasto-Plastic Model for Pressure-Dependent Yield

David Harris

March 2013

MIMS EPrint: 2013.9

Manchester Institute for Mathematical Sciences

School of Mathematics

The University of Manchester

Reports available from: http://www.manchester.ac.uk/mims/eprints

And by contacting: The MIMS Secretary

School of Mathematics

The University of Manchester

Manchester, M13 9PL, UK

ISSN 1749-9097 


\title{
A Hyperbolic Augmented Elasto-Plastic Model for Pressure-Dependent Yield
}

\author{
David Harris \\ School of Mathematics \\ University of Manchester
}

December 21, 2012

\begin{abstract}
A three-dimensional elasto-plastic model for the deformation and flow of granular materials which generalises the plastic potential model and contains an additional term analogous to that appearing in the double shearing model is presented. It is shown that for planar flows the resulting system of first order partial differential equations is hyperbolic. This is in distinct contrast to the non-associated plastic potential model rule and the double shearing model, both of which fail to be hyperbolic. The ill-posedness of the planar double shearing model is due to the presence of the rotation-rate of the principal axes of stress. The ill-posedness of the non-associated plastic potential model is due to distinct quasi-static spatial stress and velocity characteristics. The present model attains wellposedness by replacing the planar rotation-rate of the principal stress axes by the vector intrinsic spin of a Cosserat continuum and using it to ensure identical spatial stress and velocity characteristics. Flows in which the intrinsic spin vector is constant in both space and time correspond to flows in an ordinary continuum. The model governing such flows is embedded into a Cosserat model in such a way that the characteristic structure is preserved.
\end{abstract}

\section{Introduction}

A leading exponent of continuum mechanics in the 20th Century, A.J.M. Spencer, wrote 'The correct formulation of constitutive equations to describe the mechanical behaviour of granular materials on the macroscopic scale is one of the outstanding problems of continuum mechanics', Spencer (1982). Both interpretations of this sentence, namely that this is an open problem and that this is an important problem in continuum mechanics, remain true today.

In the class of elasto-plastic models, an associated flow rule is one in which the yield function also acts as the plastic potential function. This model had proved so successful in the analysis of inelastic deformations of metals, Hill 
(1950), that it seemed a natural extension to apply the same idea to soil mechanics, Drucker-Prager (1952). However, associated flow rules proved too restrictive to account for experimental properties of granular materials and attention turned to using a plastic potential function distinct from the yield function, i.e. a non-associated flow rule, Mroz et al (1979).

Broadly speaking, metals yield independently of the pressure, whereas soils and other granular materials exhibit a marked pressure dependence on yield. The kinematic quantity associated with pressure is dilatation or compaction (or their rates). The issues arising out of incorporating these two properties have proved difficult to resolve satisfactorily. An associated flow rule entails only one parameter to control the magnitude of both the pressure dependence and the dilatation. The magnitude of the dilatation is known to be rather less than that of the pressure dependence and so the model either over-estimates the dilatation or under-estimates the pressure dependence. A simple way of incorporating realistic magnitudes of the pressure dependence and dilatation is to use a non-associated flow rule which allows two independent parameters with which to model their magnitudes and this ability to satisfy experimental data has led to its frequent use in geotechnical and civil engineering. The measures of the magnitudes of pressure-dendence and of dilatation for the yield and plastic potential functions used in this paper are given by equations (42).

However, non-associated flow rules have, from the very beginning of their use, been viewed with suspicion and many arguments have been presented against their use. For example Drucker (1951) and Ilyushin (1961) introduced ad hoc postulates which lead naturally to associated flow rules. In Section 7 we also present an argument against the use of a classical non-associated flow rule, namely that non-associated flow rules are ill-posed in the context of evolutionary problems. Thus, if the inertia terms are retained in the equations of motion, then, together with the non-associated flow rule and yield condition, the solution to the Cauchy problem, as a function of time, is discontinuous in the initial data. The issue of ill-posedness in plasticity models was dealt with, in a series of papers by D.G. Schaeffer and co-workers, for example Pitman et al (1987), Schaeffer et al (1988), Schaeffer (1990) and, for the double shearing model, by Kruyt (1990). See also Harris (2001a).

The classical use of the theory is to quasi-static and time independent problems. The same property that induces ill-posedness in time-dependent problems also manifests itself in quasi-static problems and this confirms the inadequacy of non-associated flow rules. The velocity characteristic directions are distinct from the stress characteristic directions and from this it may be inferred that the domains of dependence and of influence differ. Thus, from Cauchy data on a non-characteristic boundary it follows that the stress and velocity fields are determined in overlapping, but distinct regions. This makes construction of solutions to all but the simplest problems impractical.

An alternative class of models is provided by the double sliding free rotating model, de Josselin de Jong $(1959,1977)$ and the double shearing model, Spencer $(1964,1982)$ and Mehrabadi et al (1978). The idea had previously been proposed in Mandel (1947). Single shearing models have also been proposed, 
Geniev (1958), Harris (2001b). These models are inherently planar (although they may be generalised to axially-symmetric flows of Mohr-Coulomb materials). The double sliding free rotating model introduces the crucial idea that a quantity associated with the average grain spin needs to be incorporated into continuum models of granular systems. Incorporating such a quantity into the formulation has proved very difficult to accomplish successfully. In the double sliding free rotating model the spin is governed by inequalities and is bounded in magnitude but not determined uniquely (hence the word 'free'). The inequalities are intended to express the variability of material response to a given loading. The double shearing model replaces the grain spin by the rotationrate of the principal axes of stress and there is a contribution to the velocity strain (i.e. strain-rate or deformation rate) due to the difference between the spin of the stress principal axes and the velocity spin (i.e. vorticity). The double shearing model uses the relative spin of the material and of stress to induce identical stress and velocity characteristic directions. It turns out that this class of model is also ill-posed, the double sliding free rotating model by design, the double shearing model because of the manner in which the presence of the spin of the stress principal axes interacts with the other terms of the model, see Harris (2001a). A further generalisation of the double shearing model due to Anand (1983) possesses distinct stress and velocity characterisic directions in addition to the stress rotation rate and consequently this model is ill-posed on two counts.

For further results on ill-posedness for both non-associated flow rules and the double shearing model, see Harris (2001a). A unified formulation of the double shearing and plastic potential models was obtained in Harris $(1993,1995)$, which enabled the development of a well-posed model in the context of a reduced Cosserat continuum in Harris et al (2005). The model was generalised to a truly three-dimensional constitutive equation in Harris (2006). The present paper furthers this work and presents an elasto-plastic model that incorporates pressure dependence, dilatation and the grain spin in such a way that the model is well-posed. The model may also incorporate compaction but this is not pursued here.

The model governing classical flows presented in this paper is very wellfounded experimentally. Much work has been done on fitting experimental data concerning pressure dependence of yield and dilatancy to the plastic potential model in the context of quasi-static geotechnical engineering problems. For problems involving flow the double-shearing model, in the case where the elasticity is neglected (the so-called rigid-plastic model), gives physically realistic flow patterns (more realistic than the plastic potential model with a non-associated flow rule). The present model combines the best qualities of both of these previous models and eliminates their inadequacies.

The experimental basis for the use of Cosserat models is much less clear cut. A classical continuum provides no internal length scale for the material and localisation leads to shear bands of zero width, i.e. reduces to discontinuities in some of the dependent variables. In real granular materials, shear bands are of several grain diameters in width and a Cosserat continuum does admit 
an internal length scale which may be used to account for this. However, the complexity of Cosserat models and the paucity of experimentally verified effects makes the construction of mathematical models, which are both simple and do not incorporate superfluous effects, problematic. The model presented here for flows which are describable by an ordinary continuum contains degenerate values of a Cosserat quantity. It is therefore necessary, in order to complete the modelling process, to embed the model for such flows into a Cosserat model. In this paper we embed the model into the simplest possible Cosserat model which will enable the characteristic structure of the classical model to be preserved. This provides a base Cosserat model from which to develop more complicated models to account for those experimental properties of granular materials which ultimately may be ascribed to Cosserat effects (this effectively means to finite grain-size effects).

\section{Mathematical Formulation}

Relative to an inertial frame of reference, take rectangular Cartesian coordinate axes $O x_{i}$ and denote the position vector of a point $P$ relative to $O$ by $\mathbf{x}$. Let $\boldsymbol{\sigma}$ denote the Cauchy stress tensor, $\rho$ the bulk density of the material, $\mathbf{v}$ the Eulerian velocity vector, $d_{t}$ the convected derivative, $\partial_{i}$ the partial derivative with respect to $x_{i}$ and $\partial_{t}$ (or $\partial_{0}$ ) the partial derivative with respect to time $t$. Let $\gamma=\left(\gamma_{i j}\right)$ denote the velocity gradient tensor and let $\mathbf{d}$, s denote the symmetric and anti-symmetric parts of $\gamma$,

$$
\mathbf{d}=\frac{1}{2}\left(\gamma+\gamma^{T}\right), \quad \mathbf{s}=\frac{1}{2}\left(\gamma-\gamma^{T}\right)
$$

and call $\mathbf{d}$ the velocity strain and $\mathbf{s}$ the velocity spin.

The model for a standard continuum that is presented here is formulated in terms of a Cosserat continuum (see Section 10) in which the Cosserat quantities are either identically zero or everywhere constant. One Cosserat quantity appears in the constitutive equation for a standard continuum but takes a degenerate value (namely a constant, which is zero relative to certain frames, in both space and time). In addition to the Eulerian velocity $\mathbf{v}$ we shall suppose that, at each point $P$, an Eulerian vector quantity called the intrinsic spin, denoted by $\boldsymbol{\Omega}^{v}=\left(\Omega_{i}\right)$, is defined. This is a primitive quantity and, as such, needs no definition. The intuitive interpretations that we give to $\mathbf{v}$ and $\boldsymbol{\Omega}^{v}$ are as follows. In the real granular material, or in a discrete mathematical representation of it, take a representative volume element (RVE) comprising $N$ grains about each point $P$ in the granular material. The size and shape of the RVE need not be the same for every point, for example for points interior to a shear band the RVE may be chosen differently from the RVE for points exterior to the shear band. Let $M$ denote the total mass of the grains interior to the RVE then we may identify $\mathbf{v}$ at the point $P$ with the total momentum of the grains in

the RVE divided by $M$. Similarly, let $\mathbf{I}$ denote the moment of inertia tensor for all the grains in the RVE then we identify $\boldsymbol{\Omega}$ with the total angular momentum 
of the grains in the RVE multiplied by the inverse of the moment of inertia tensor. The anti-symmetric tensor dual to $\boldsymbol{\Omega}^{v}$, called the intrinsic spin tensor, and denoted by $\Omega=\left(\Omega_{i j}\right)$ is defined by

$$
\boldsymbol{\Omega}=-\varepsilon \cdot \boldsymbol{\Omega}^{v}, \quad \Omega_{i j}=-\varepsilon_{i j k} \Omega_{k}^{v}
$$

where $\varepsilon$ denotes the permutation tensor. The relative spin, $\boldsymbol{\omega}$, is defined as the difference between the velocity spin and the intrinsic spin,

$$
\omega=\mathrm{s}-\Omega \text {. }
$$

Note that $\boldsymbol{\omega}$ is frame-indifferent, being the difference in two spins, and hence may appear in constitutive equations. We regard $\boldsymbol{\omega}$ as a fundamental quantity required for the kinematic description of granular materials. Deformations and flows in which the intrinsic spin $\boldsymbol{\Omega}$ is constant everywhere at all times and in which all other Cosserat quantities are identically zero will be called a classical deformation or flow. The constant value of $\boldsymbol{\Omega}$ may always be taken to be zero by a suitable choice of reference frame.

\section{Equations Governing Classical Deformations and Flows}

The equations governing classical flows are as follows and are written in both direct and indicial notation (using the double suffix summation convention). The balance laws are

(a) Cauchy's equations of translational motion

$$
\nabla \cdot \boldsymbol{\sigma}+\rho \mathbf{F}=\rho d_{t} \mathbf{v}, \quad \partial_{j} \sigma_{j i}+\rho F_{i}=\rho d_{t} v_{i} .
$$

where $\mathbf{F}$ denotes the body force.

(b) Continuity of mass

$$
d_{t} \rho+\rho \nabla \cdot \mathbf{v}=0, \quad d_{t} \rho+\rho \partial_{j} v_{j}=0 .
$$

The constitutive equations comprise a yield criterion and a flow rule. The existence is assumed of two scalar valued functions of the stress, the yield function $f$ and the plastic potential function $g$ (this assumption can be weakened but it simplifies the analysis).

(c) Yield condition

$$
f(\boldsymbol{\sigma}) \leq 0
$$

where $f$ denotes a scalar valued function of the stress $\boldsymbol{\sigma}$. At a point $P$ such that $f(\boldsymbol{\sigma})<0$, the material is said to be in an elastic state. At a point $P$ such that $f(\boldsymbol{\sigma})=0$, the material is said to be in a state of yield or in a plastic state. For the yield function $f(\boldsymbol{\sigma})$ and plastic potential function $g(\boldsymbol{\sigma})$ define the second order tensors $\mathbf{f}$ and $\mathbf{g}$ by

$$
\mathbf{f}=\left(f_{i j}\right)=\frac{\partial f}{\partial \boldsymbol{\sigma}}=\left(\frac{\partial f}{\partial \sigma_{i j}}\right), \quad \mathbf{g}=\left(g_{i j}\right)=\frac{\partial g}{\partial \boldsymbol{\sigma}}=\left(\frac{\partial g}{\partial \sigma_{i j}}\right),
$$


and call them the yield and flow tensors, respectively. The equation of continuing yield reads

$$
\mathbf{f} \cdot d_{t} \boldsymbol{\sigma}=0, \quad f_{i j} d_{t} \sigma_{i j}=0 .
$$

(d) The flow rule is the elasto-plastic flow rule augmented by a term analogous to a term of the double shearing model, namely

$$
\mathbf{d}=\left\{\begin{array}{ccc}
\mathbf{M}: \stackrel{\circ}{\boldsymbol{\sigma}}+\lambda \mathbf{g}+\mu(\boldsymbol{\omega} \cdot \boldsymbol{\sigma}-\boldsymbol{\sigma} \cdot \boldsymbol{\omega}) & \text { if } & f(\boldsymbol{\sigma})=0 \\
\mathbf{M}: \stackrel{\circ}{\boldsymbol{\sigma}} & \text { if } & f(\boldsymbol{\sigma})<0
\end{array}\right.
$$

where $\mathbf{M}$ denotes the 4 th order elastic compliance tensor, $\lambda$ is an arbitrary non-negative scalar multiplier and $\mu$ is called the relative spin coefficient. It is important to note that the scalar quantity $\mu$ is not to be regarded as a disposable material parameter. Its value will be determined by what we regard as a physical law in Section 6 , see equ.(47). The term $\boldsymbol{\omega} \cdot \boldsymbol{\sigma}-\boldsymbol{\sigma} \cdot \boldsymbol{\omega}$ may be intrerpreted physically as the difference in the stress rate as measured in two frames, one spinning with the vorticity, the other fixed relative to the intrinsic spin $\boldsymbol{\Omega}$. See Harris et al (2005) for a derivation of the plastic part of the model in the planar case. A statement of the three-dimensional model was first given in Harris (2006). Note that if $\mu=0$, the quantity $\boldsymbol{\omega}$ no longer appears in equ. (9) which reduces to the equations governing the plastic potential model.

This completes the statement of the model and we now consider a planar deformation in the $O x_{1} x_{2}$ plane in which the $O x_{3}$ direction is a principal stress direction. We shall restrict attention to perfect plasticity (i.e. no strain-, workor density-hardening or softening). The equations governing such deformations and flows are as follows. The planar balance of linear momentum equations are

$$
\rho d_{t} v_{1}-\partial_{1} \sigma_{11}-\partial \sigma_{12}-F_{1} \rho=0, \quad \rho d_{t} v_{2}-\partial_{1} \sigma_{12}-\partial_{2} \sigma_{22}-F_{2} \rho=0,
$$

where

$$
d_{t}=\partial_{t}+v_{1} \partial_{1}+v_{2} \partial_{2}
$$

and the balance of rotational momentum reduces to $\sigma_{12}=\sigma_{21}$. The equation of continuing yield is

$$
f_{11} d_{t} \sigma_{11}+2 f_{12} d_{t} \sigma_{12}+f_{22} d_{t} \sigma_{22}=0
$$

The planar continuity of mass equation is

$$
d_{t} \rho+\rho\left(\partial_{1} v_{1}+\partial_{2} v_{2}\right)=0
$$

We shall assume elastic isotropy and define

$$
a=E^{-1}\left(1-\nu^{2}\right), \quad b=-E^{-1}(1+\nu) \nu, \quad c=2 E^{-1}(1+\nu)
$$

where $E$ denotes Young's modulus and $\nu$ Poisson's ratio. Let

$$
\delta_{1}=(\mu-c / 2) \sigma_{12}, \quad \delta_{2}=(\mu-c / 2)\left(\sigma_{11}-\sigma_{22}\right)
$$


then the planar flow rule becomes

$$
\begin{gathered}
\left(-\partial_{1}+\delta_{1} \partial_{2}\right) v_{1}-\delta_{1} \partial_{1} v_{2}+a d_{t} \sigma_{11}+b d_{t} \sigma_{22}+g_{11} \lambda+2 \mu \Omega_{3} \sigma_{12}=0, \\
-\left(1+\delta_{2}\right) \partial_{2} v_{1}+\left(-1+\delta_{2}\right) \partial_{1} v_{2}+c d_{t} \sigma_{12}+2 g_{12} \lambda-2 \mu \Omega_{3}\left(\sigma_{11}-\sigma_{22}\right)=0 \\
-\delta_{1} \partial_{2} v_{1}+\left(-\partial_{2}+\delta_{1} \partial_{1}\right) v_{2}+b d_{t} \sigma_{11}+a d_{t} \sigma_{22}+g_{22} \lambda-2 \mu \Omega_{3} \sigma_{12}=0
\end{gathered}
$$

Let $\mathbf{z}$ denote the vector of dependent quantities given by

$$
\mathbf{z}^{T}=\left(v_{1}, v_{2}, \lambda, \sigma_{11}, \sigma_{12}, \sigma_{22}, \rho\right),
$$

and let

$$
\mathbf{b}^{T}=\left(0,0,0,2 \mu \Omega_{3} \sigma_{12},-2 \mu \Omega_{3} \sigma_{12},-2 \mu \Omega_{3}\left(\sigma_{11}-\sigma_{22}\right), 0\right)
$$

where superscipt $T$ denotes transpose Let $A$ denote the matrix

$$
A=\left[\begin{array}{ccccccc}
\rho d_{t} & 0 & 0 & -\partial_{1} & -\partial_{2} & 0 & -F_{1} \\
0 & \rho d_{t} & 0 & 0 & -\partial_{1} & -\partial_{2} & -F_{2} \\
0 & 0 & 0 & f_{11} d_{t} & f_{12} d_{t} & f_{22} d_{t} & 0 \\
-\partial_{1}+\delta_{1} \partial_{2} & -\delta_{1} \partial_{1} & g_{11} & a d_{t} & 0 & b d_{t} & 0 \\
-\left(1+\delta_{2}\right) \partial_{2} & \left(-1+\delta_{2}\right) \partial_{1} & 2 g_{12} & 0 & c d_{t} & 0 & 0 \\
-\delta_{1} \partial_{2} & -\partial_{2}+\delta_{1} \partial_{1} & g_{22} & b d_{t} & 0 & a d_{t} & 0 \\
\rho \partial_{1} & \rho \partial_{2} & 0 & 0 & 0 & 0 & d_{t}
\end{array}\right]
$$

then in matrix form the equations governing the model are

$$
A \mathbf{z}+\mathbf{b}=\mathbf{0} .
$$

\section{Linearisation of the Equations Governing Clas- sical Flows}

Equ. (22) represents a system of first order partial differential equations for the quantities $\mathbf{z}^{T}$. Let

$$
\mathbf{Z}^{T}=\left(V_{1}, V_{2}, \Lambda, \Sigma_{11}, \Sigma_{12}, \Sigma_{22}, \varrho\right), \quad \mathbf{z}^{\prime T}=\left(v_{1}^{\prime}, v_{2}^{\prime}, \lambda^{\prime}, \sigma_{11}^{\prime}, \sigma_{12}^{\prime}, \sigma_{22}^{\prime}, \rho^{\prime}\right)
$$

denote a given solution of equ. (22) and an arbitrary perturbation of this given solution, respectively. Let $\mathbf{z}=\mathbf{Z}+\mathbf{z}^{\prime}$, i.e.

$$
\begin{gathered}
v_{1}=V_{1}+v_{1}^{\prime}, \quad v_{2}=V_{2}+v_{2}^{\prime}, \quad \dot{\lambda}=\dot{\Lambda}+\dot{\lambda}^{\prime}, \quad \sigma_{11}=\Sigma_{11}+\sigma_{11}^{\prime}, \\
\sigma_{12}=\Sigma_{12}+\sigma_{12}^{\prime}, \quad \sigma_{22}=\Sigma_{22}+\sigma_{22}^{\prime}, \quad \rho=\varrho+\rho^{\prime} .
\end{gathered}
$$

In this section the perturbed solution is also assumed to be a classical flow. For the case in which the perturbed solution may involve non-trivial values of Cosserat quantities, see Section 11. Linearising equ. (22) and using the fact that $\mathbf{Z}$ is a solution of equ. (22) gives the following system of linear pdes for 
$\mathbf{z}^{\prime}$. Let $c_{i j}$ denote those coefficients of components of $\mathbf{z}^{\prime}$ evaluated in the given solution $\mathbf{Z}$ which are of zeroth order in derivatives of components of $\mathbf{z}^{\prime}$, where $i$ denotes the equation and $j$ the component $\mathbf{z}^{\prime}$.

(a) First component of the equation of linear momentum

$$
\left(\varrho D_{t}+c_{11}\right) v_{1}^{\prime}+c_{12} v_{2}^{\prime}-\partial_{1} \sigma_{11}^{\prime}-\partial_{2} \sigma_{12}^{\prime}+c_{17} \rho^{\prime}=0,
$$

where

$$
D_{t}=\partial_{1}+V_{1} \partial_{1}+V_{2} \partial_{2}, \quad c_{11}=\varrho \Gamma_{11}, \quad c_{12}=\varrho \Gamma_{12}, \quad c_{17}=D_{t} V_{1}-F_{1}
$$

and $\Gamma_{i j}=\partial V_{i} / \partial x_{j}$.

(b) Second component of the equation of linear momentum

$$
c_{21} v_{1}^{\prime}+\left(\varrho D_{t}+c_{22}\right) v_{2}^{\prime}-\partial_{1} \sigma_{12}^{\prime}-\partial_{2} \sigma_{22}^{\prime}+c_{27} \rho^{\prime}=0,
$$

where

$$
c_{21}=\varrho \Gamma_{21}, \quad c_{22}=\varrho \Gamma_{22}, \quad c_{27}=D_{t} V_{2}-F_{2} .
$$

(c) Continuing yield

Assuming that $f(\boldsymbol{\sigma})$ is twice differentiable with respect to its arguments, let

$$
F_{i j}=f_{i j}\left(\Sigma_{11}, \Sigma_{12}, \Sigma_{22}\right), \quad F_{i j k l}=\left.\frac{\partial f_{i j}}{\partial \sigma_{k l}}\right|_{\Sigma},
$$

then

$$
\left(F_{11} D_{t}+c_{34}\right) \sigma_{11}^{\prime}+\left(2 F_{12} D_{t}+c_{35}\right) \sigma_{12}^{\prime}+\left(F_{22} D_{t}+c_{36}\right) \sigma_{22}^{\prime}=0 .
$$

where

$$
\begin{aligned}
& c_{34}=F_{1111} D_{t} \Sigma_{11}+2 F_{1211} D_{t} \Sigma_{12}+F_{2211} D_{t} \Sigma_{22}, \\
& c_{35}=F_{1112} D_{t} \Sigma_{11}+2 F_{1212} D_{t} \Sigma_{12}+F_{2212} D_{t} \Sigma_{22}, \\
& c_{36}=F_{1122} D_{t} \Sigma_{11}+2 F_{1222} D_{t} \Sigma_{12}+F_{2222} D_{t} \Sigma_{22} .
\end{aligned}
$$

Now let

$$
\Delta_{1}=(\mu-c / 2) \Sigma_{12}, \quad \Delta_{2}=(\mu-c / 2)\left(\Sigma_{11}-\Sigma_{22}\right) .
$$

(d) The first component of the flow rule is

$$
\begin{aligned}
& \left(-\partial_{1}+\Delta_{1} \partial_{2}+c_{41}\right) v_{1}^{\prime}+\left(-\Delta_{1} \partial_{1}+c_{42}\right) v_{2}^{\prime}+G_{11} \lambda^{\prime} \\
& +\left(a D_{t}+c_{44}\right) \sigma_{11}^{\prime}+c_{45} \sigma_{12}^{\prime s}+\left(b D_{t}+c_{46}\right) \sigma_{22}^{\prime}=0
\end{aligned}
$$

where

$$
\begin{aligned}
& c_{41}=a \partial_{1} \Sigma_{11}+b \partial_{1} \Sigma_{22}, \quad c_{42}=a \partial_{2} \Sigma_{11}+b \partial_{2} \Sigma_{22}, \\
& c_{44}=c S_{21}-2 \mu\left(S_{21}-\Omega_{3}\right)+\Lambda G_{1112}, \quad c_{45}=\Lambda G_{1111}, \quad c_{46}=\Lambda G_{1122},
\end{aligned}
$$

where

$$
G_{i j}=g_{i j}\left(\Sigma_{11}, \Sigma_{12}, \Sigma_{22}\right), \quad G_{i j k l}=\left.\frac{\partial g_{i j}}{\partial \sigma_{k l}}\right|_{\Sigma} .
$$


(e) The second component of the flow rule is

$$
\begin{gathered}
{\left[-\left(1+\Delta_{2}\right) \partial_{2}+c_{51}\right] v_{1}^{\prime}+\left[\left(-1+\Delta_{2}\right) \partial_{1}+c_{52}\right] v_{2}^{\prime}+2 G_{12} \lambda^{\prime}} \\
+c_{54} \sigma_{11}^{\prime}+\left(c D_{t}+c_{55}\right) \sigma_{12}^{\prime}+c_{56} \sigma_{22}^{\prime}=0
\end{gathered}
$$

where

$$
\begin{aligned}
& c_{51}=c \partial_{1} \Sigma_{12}, \quad c_{52}=c \partial_{2} \Sigma_{12}, \quad c_{54}=2 \mu\left(S_{21}-\Omega_{3}\right)-c S_{21}+2 \Lambda G_{1211} \\
& c_{55}=2 \Lambda G_{1212}, \quad c_{56}=c S_{21}-2 \mu\left(S_{21}-\Omega_{3}\right)+2 \Lambda G_{1222} .
\end{aligned}
$$

(f) The third component of the flow rule is

$$
\begin{aligned}
& \left(-\Delta_{1} \partial_{2}+c_{61}\right) v_{1}^{\prime}+\left(-\partial_{2}+\Delta_{1} \partial_{1}+c_{62}\right) v_{2}^{\prime}+G_{22} \lambda^{\prime} \\
& +\left(b D_{t}+c_{64}\right) \sigma_{11}^{\prime}+c_{65} \sigma_{12}^{\prime}+\left(a D_{t}+c_{66}\right) \sigma_{22}^{\prime}=0
\end{aligned}
$$

where

$$
\begin{aligned}
& c_{61}=b \partial_{1} \Sigma_{11}+a \partial_{1} \Sigma_{22}, \quad c_{62}=b \partial_{2} \Sigma_{11}+a \partial_{2} \Sigma_{22}, \\
& c_{64}=\Lambda G_{2211}, \quad c_{65}=2 \mu\left(S_{21}-\Omega_{3}\right)-c S_{21}+\Lambda G_{2212}, \quad c_{66}=\Lambda G_{2222} .
\end{aligned}
$$

(g) Mass continuity

$$
\left(\varrho \partial_{1}+c_{71}\right) v_{1}^{\prime}+\left(\varrho \partial_{2}+c_{72}\right) v_{2}^{\prime}+\left(D_{t}+c_{77}\right) \rho^{\prime}=0,
$$

where

$$
c_{71}=\partial_{1} \varrho, \quad c_{72}=\partial_{2} \varrho, \quad c_{77}=\Gamma_{11}+\Gamma_{22} .
$$

\section{Characteristic Equation Governing Classical Flows}

The linearised equations may be written in matrix form as

$$
A^{\prime} \mathbf{z}^{\prime}=\mathbf{0}
$$

where

$$
A^{\prime}=\left[\begin{array}{ccccccc}
a_{11} & c_{12} & 0 & a_{14} & a_{15} & 0 & c_{17} \\
c_{21} & a_{22} & 0 & 0 & a_{25} & a_{26} & c_{27} \\
0 & 0 & 0 & a_{34} & a_{35} & a_{36} & 0 \\
b_{41} & b_{42} & G_{11} & b_{44} & c_{45} & b_{46} & 0 \\
b_{51} & b_{52} & 2 G_{12} & c_{54} & b_{55} & c_{56} & 0 \\
b_{61} & b_{62} & G_{22} & b_{64} & c_{65} & b_{66} & 0 \\
d_{71} & d_{72} & 0 & 0 & 0 & 0 & d_{77}
\end{array}\right]
$$

where

$$
a_{11}=\varrho D_{t}+c_{11}, \quad a_{14}=-\partial_{1}, \quad a_{15}=-\partial_{2},
$$




$$
\begin{gathered}
a_{22}=\varrho D_{t}+c_{22}, \quad a_{25}=-\partial_{1}, \quad a_{26}=-\partial_{2}, \\
a_{34}=F_{11} D_{t}+c_{34}, \quad a_{35}=2 F_{12} D_{t}+c_{35}, \quad a_{36}=F_{22} D_{t}+c_{36}, \\
b_{41}=\left(-\partial_{1}+\Delta_{1} \partial_{2}\right)+c_{41}, \quad b_{42}=-\Delta_{1} \partial_{1}+c_{42}, \quad b_{44}=a D_{t}+c_{44}, \quad b_{46}=b D_{t}+c_{46}, \\
b_{51}=-\left(1+\Delta_{2}\right) \partial_{2}+c_{51}, \quad b_{52}=\left(-1+\Delta_{2}\right) \partial_{1}+c_{52}, \quad b_{55}=c D_{t}+c_{55}, \\
b_{61}=-\Delta_{1} \partial_{2}+c_{61}, \quad b_{62}=-\partial_{2}+\Delta_{1} \partial_{1}+c_{62}, \quad b_{64}=b D_{t}+c_{64}, \quad b_{66}=a D_{t}+c_{66}, \\
d_{71}=\varrho \partial_{1}+c_{71}, \quad d_{72}=\varrho \partial_{2}+c_{72}, \quad d_{77}=D_{t}+c_{77} .
\end{gathered}
$$

Let the matrix $A^{s}$ be obtained from $A^{\prime}$ by replacing every occurence of $\partial_{k}$, where $k=0,1,2$ by the algebraic quantity $i \xi_{k}$, where $i=\sqrt{-1}$. The symbol of the system of pdes is the determinant of $A^{s}$. Let $\bar{\xi}=\xi_{0}+V_{1} \xi_{1}+V_{2} \xi_{2}$ then the symbol is

$$
\operatorname{det} A^{s}=\left[\begin{array}{ccccccc}
a_{11}^{s} & c_{12} & 0 & a_{14}^{s} & a_{15}^{s} & 0 & c_{17} \\
c_{21} & a_{22}^{s} & 0 & 0 & a_{25}^{s} & a_{26}^{s} & c_{27} \\
0 & 0 & 0 & a_{34}^{s} & a_{35}^{s} & a_{36}^{s} & 0 \\
b_{41}^{s} & b_{42}^{s} & G_{11} & b_{44}^{s} & c_{45} & b_{46}^{s} & 0 \\
b_{51}^{s} & b_{52}^{s} & 2 G_{12} & c_{54} & b_{55}^{s} & c_{56} & 0 \\
b_{61}^{s} & b_{62}^{s} & G_{22} & b_{64}^{s} & c_{65} & b_{66}^{s} & 0 \\
d_{71}^{s} & d_{72}^{s} & 0 & 0 & 0 & 0 & d_{77}^{s}
\end{array}\right]
$$

where

$$
\begin{gathered}
a_{11}^{s}=\varrho i \bar{\xi}+c_{11}, \quad a_{14}^{s}=-i \xi_{1}, \quad a_{15}^{s}=-i \xi_{2}, \\
a_{22}^{s}=\varrho i \bar{\xi}+c_{22}, \quad a_{25}^{s}=-i \xi_{1}, \quad a_{26}^{s}=-i \xi_{2}, \\
a_{34}^{s}=F_{11} i \bar{\xi}+c_{34}, \quad a_{35}^{s}=2 F_{12} i \bar{\xi}+c_{35}, \quad a_{36}^{s}=F_{22} i \bar{\xi}+c_{36}, \\
b_{41}^{s}=\left(-i \xi_{1}+\Delta_{1} i \xi_{2}\right)+c_{41}, \quad b_{42}^{s}=-\Delta_{1} i \xi_{1}+c_{42}, \quad b_{44}^{s}=a i \bar{\xi}+c_{44}, \quad b_{46}^{s}=b i \bar{\xi}+c_{46}, \\
b_{51}^{s}=-\left(1+\Delta_{2}\right) i \xi_{2}+c_{51}, \quad b_{52}^{s}=\left(-1+\Delta_{2}\right) i \xi_{1}+c_{52}, \quad b_{55}^{s}=c i \bar{\xi}+c_{55}, \\
b_{61}^{s}=-\Delta_{1} i \xi_{2}+c_{61}, \quad b_{62}^{s}=\left(-i \xi_{2}+\Delta_{1} i \xi_{1}\right)+c_{62}, \quad b_{64}^{s}=b i \bar{\xi}+c_{64}, \quad b_{66}^{s}=a i \bar{\xi}+c_{66}, \\
d_{71}^{s}=\varrho i \xi_{1}+c_{71}, \quad d_{72}^{s}=\varrho i \xi_{2}+c_{72}, \quad d_{77}^{s}=i \bar{\xi}+c_{77} .
\end{gathered}
$$

The principal part of the symbol is the homogeneous polynomial comprising all highest order terms in $\xi_{k}$ and provides an efficient method to determine the type of the system of pdes. Every term in the expansion of $\operatorname{det} A^{s}$ which contains a term $c_{i j}$ is a lower order term in the $\xi_{k}$. Let the matrix $A^{p}$ be obtained from $A^{s}$ by replacing every $c_{i j}$ in $A^{s}$ by zero, i.e.

$$
A^{p}=\left[\begin{array}{ccccccc}
\varrho i \bar{\xi} & 0 & 0 & -i \xi_{1} & -i \xi_{2} & 0 & 0 \\
0 & \varrho i \bar{\xi} & 0 & 0 & -i \xi_{1} & -i \xi_{2} & 0 \\
0 & 0 & 0 & F_{11} i \bar{\xi} & 2 F_{12} i \bar{\xi} & F_{22} i \bar{\xi} & 0 \\
-i \xi_{1}+\Delta_{1} i \xi_{2} & -\Delta_{1} i \xi_{1} & G_{11} & a i \bar{\xi} & 0 & b i \bar{\xi} & 0 \\
-\left(1+\Delta_{2}\right) i \xi_{2} & \left(-1+\Delta_{2}\right) i \xi_{1} & 2 G_{12} & 0 & c i \bar{\xi} & 0 & 0 \\
-\Delta_{1} i \xi_{2} & \left(-i \xi_{2}+\Delta_{1} i \xi_{1}\right) & G_{22} & b i \bar{\xi} & 0 & a i \bar{\xi} & 0 \\
\varrho i \xi_{1} & \varrho i \xi_{2} & 0 & 0 & 0 & 0 & i \bar{\xi}
\end{array}\right] .
$$


The principal part of the symbol is obtained by expanding the determinant of the matrix $A^{p}$ and this gives either $\bar{\xi}^{2}=0$ or

$$
\begin{gathered}
\varrho^{2} A \bar{\xi}^{4}+\varrho\left(B \xi_{1}^{2}+C \xi_{1} \xi_{2}+D \xi_{2}^{2}\right) \bar{\xi}^{2} \\
+\left(F_{22} \xi_{1}^{2}-2 F_{12} \xi_{1} \xi_{2}+F_{11} \xi_{2}^{2}\right)\left(H_{22} \xi_{1}^{2}-2 H_{12} \xi_{1} \xi_{2}+H_{11} \xi_{2}^{2}\right)=0
\end{gathered}
$$

where

$$
\begin{aligned}
& A=4\left(a^{2}-b^{2}\right) F_{12} G_{12}+c\left[a\left(F_{11} G_{11}+F_{22} G_{22}\right)-b\left(F_{11} G_{22}+F_{22} G_{11}\right)\right], \\
& B=-a\left(F_{11} G_{11}+4 F_{12} G_{12}+F_{22} G_{22}\right)+b\left(F_{11} G_{22}+F_{22} G_{11}\right)-c F_{22} G_{22} \\
&-2(a+b) \Delta_{1}\left(F_{22}-F_{11}\right) G_{12} \\
&+a \Delta_{2}\left(F_{11} G_{11}+F_{22} G_{22}\right)-b \Delta_{2}\left(F_{11} G_{22}+F_{22} G_{11}\right), \\
& C= 2(a-b)\left[\left(F_{11}+F_{22}\right) G_{12}+F_{12}\left(G_{11}+G_{22}\right)\right]+8(a+b) \Delta_{1} F_{12} G_{12} \\
&+c \Delta_{1}\left(F_{11}+F_{22}\right)\left(G_{11}+G_{22}\right)-2(a+b) \Delta_{2} F_{12}\left(G_{22}-G_{11}\right), \\
&-a\left(F_{11} G_{11}+4 F_{12} G_{12}+F_{22} G_{22}\right)+b\left(F_{11} G_{22}+F_{22} G_{11}\right)-c F_{11} G_{11} \\
&+2(a+b) \Delta_{1}\left(F_{22}-F_{11}\right) G_{12} \\
&-a \Delta_{2}\left(F_{11} G_{11}+F_{22} G_{22}\right)+b \Delta_{2}\left(F_{11} G_{22}+F_{22} G_{11}\right), \\
& H_{22}=\left(1-\Delta_{2}\right) G_{22}+2 \Delta_{1} G_{12}, \quad H_{12}=G_{12}+\Delta_{1}\left(G_{11}+G_{22}\right), \\
& H_{11}=\left(1+\Delta_{2}\right) G_{11}+2 \Delta_{1} G_{12} .
\end{aligned}
$$

Notes: (i) The system of pdes, equ. (34), is of order seven and gives rise to a characteristic equation of degree six (and so the system is degenerate) which has a repeated linear factor

$$
\xi_{0}+V_{1} \xi_{1}+V_{2} \xi_{2}=0
$$

(ii) The remaining factor, equ. (36), is a homogeneous polynomial of degree four in $\bar{\xi}, \xi_{1}, \xi_{2}$ such that every term in the coefficients contains exactly one $F_{i j}$ and exactly one $G_{i j}$. Equ. (36) is investigated thoroughly in Sections 6 - 9.

(iii) The terms independent of $\bar{\xi}$ (which corresponds to the quasi-static case) factorise in such a way that the contributions from the stress and velocity uncouple. The roots of both factors are real and distinct provided that

$$
F_{12}^{2}-F_{11} F_{22}>0, \quad H_{12}^{2}-H_{11} H_{22}>0 .
$$

We shall suppose these restrictions on $f$ and $g$ to hold true. 


\section{Transformation of the Characteristic Equation}

Define the planar invariants

$$
p=-\frac{1}{2}\left(\sigma_{11}+\sigma_{22}\right), \quad q=\frac{1}{2}\left[\left(\sigma_{11}-\sigma_{22}\right)^{2}+4 \sigma_{12}^{2}\right]^{1 / 2}
$$

then the in plane components of the stress tensor may be written

$$
\sigma_{11}=-p+q \cos 2 \psi, \quad \sigma_{22}=-p-q \cos 2 \psi, \quad \sigma_{12}=q \sin 2 \psi
$$

where $\psi$ denotes the angle that the greater principal stress makes with the positive $x$-axis,

$$
\tan 2 \psi=\frac{2 \sigma_{12}}{\sigma_{11}-\sigma_{22}} .
$$

So far we have not assumed plastic isotropy and equ. (36) holds for plastic anisotropy (but elastic isotropy has been assumed). At this point we now assume plastic isotropy. We also now make the assumption that the material obeys a general Mohr-Coulomb type yield condition, i.e. we assume that

$$
f=f(p, q)
$$

and define the angle of internal friction and the angle of dilatancy by

$$
\sin \phi=-\frac{f_{p}}{f_{q}}, \quad \sin \nu_{p}=-\frac{g_{p}}{g_{q}}
$$

respectively, where the subscripts $p$ and $q$ denote partial differentiation with respect to that variable and $0 \leq \phi<\pi / 2,0 \leq \nu_{p}<\pi / 2$. The restriction on $f$ given in the first inequality (37) is equivalent to $\left|f_{p} / f_{q}\right| \leq 1$ and under this condition the first definition in equ. (42) makes sense. If this condition is satisfied then $g$ will automatically satisfy $\left|g_{p} / g_{q}\right| \leq 1$, since the condition of non-negative work-rate imposes the restriction that $\nu_{p} \leq \phi$ and we shall always assume this to be the case. Then we may write

$$
\begin{gathered}
F_{11}=\frac{1}{2} F_{q}(\sin \phi+\cos 2 \Psi), \quad F_{22}=\frac{1}{2} F_{q}(\sin \phi-\cos 2 \Psi), \\
F_{12}=\frac{1}{2} F_{q} \sin 2 \Psi, \\
G_{11}=\frac{1}{2} G_{q}\left(\sin \nu_{p}+\cos 2 \Psi\right), \quad G_{22}=\frac{1}{2} G_{q}\left(\sin \nu_{p}-\cos 2 \Psi\right), \\
G_{12}=\frac{1}{2} G_{q} \sin 2 \Psi,
\end{gathered}
$$

where upper-case letters denote that the quantity is evaluated in the underlying solution Z. Note (iii) after eq. (36) states that the quasi-static characteristic equation uncouples the contributions from the stress and velocity and, in the event of the roots being real and distinct, there are two stress and two velocity 
characteristic directions. The stress characteristics directions will be referred to as yield directions and the velocity characteristic directions will be referred to as slip directions. It is implicit in eq. (36) that if the stress and velocity characteristics do not coincide (i.e. the yield and slip directions do not coincide) then eq. (36) will have complex roots (see section 7 where an example of this is worked out). Another example of this situation is the model proposed in Anand (1983). We regard it as a fundamental physical law that the yield and slip directions coincide and assume henceforth that this condition is satisfied. It enables us to calculate the value of $\mu$ in equ. (9) in the case of planar flows. A sufficient condition for coincident quasi-static stress and velocity characteristic directions is that the $H_{i j}=k F_{i j}$ for some $k$. This is accomplished if the following condition is satisfied

$$
2 \mu q=\frac{q}{E}(1+\nu)+\frac{\sin \phi-\sin \nu_{p}}{1-\sin \nu_{p} \sin \phi} .
$$

and we regard this as an equation to determine $\mu$. Note that the first term on the right hand side is due to the retention of the rotational terms in the Jaumann derivative of the stress rate in the elastic law. Since the quantity $q / E$ is small this term is often neglected. Indeed, for an associated rule, it must be neglected in order to obtain a well-posed model. For the model proposed here, it may be either retained or neglected. Let

$$
\begin{gathered}
A_{1}=\cos ^{2} \nu_{p} \geq 0, \\
B_{1}=\left(1-\nu+\sin \phi \sin \nu_{p}\right)\left(1-\sin \nu_{p} \sin \phi\right) \geq 0, \\
C_{1}=\cos ^{2} \nu_{p} \sin \phi-\left(\sin \phi-\sin \nu_{p}\right) \nu, \\
D_{1}=\left(1-\sin \nu_{p} \sin \phi\right)\left(1-2 \nu+\sin \phi \sin \nu_{p}\right) \geq 0,
\end{gathered}
$$

then using equ. (43) - (47), the characteristic equation (36) becomes

$$
\begin{gathered}
A_{1}\left[(\sin \phi-\cos 2 \Psi) \xi_{1}^{2}-2 \sin 2 \Psi \xi_{1} \xi_{2}+(\sin \phi+\cos 2 \Psi) \xi_{2}^{2}\right]^{2} \\
-4\left\{\left(B_{1}-C_{1} \cos 2 \Psi\right) \xi_{1}^{2}-2 C_{1} \sin 2 \Psi \xi_{1} \xi_{2}+\left(B_{1}+C_{1} \cos 2 \Psi\right) \xi_{2}^{2}\right\} E^{-1}(1+\nu) \rho \bar{\xi}^{2} \\
+4 D_{1}\left[E^{-1}(1+\nu) \rho \bar{\xi}^{2}\right]^{2}=0
\end{gathered}
$$

Define

$$
\xi=\sqrt{\xi_{1}^{2}+\xi_{2}^{2}}, \quad \tan \theta=\frac{\xi_{2}}{\xi_{1}}, \quad m=\sqrt{\frac{1+\nu}{E}} \rho \frac{\bar{\xi}}{\xi}
$$

then the polar form of the characteristic equation is

$$
A_{1}[\sin \phi-\cos 2(\Psi-\theta)]^{2}-4\left[B_{1}-C_{1} \cos 2(\Psi-\theta)\right] m^{2}+4 D_{1} m^{4}=0 .
$$

The physical interpretation of $m$ and $\theta$ is that a plane wave in the direction $(\cos \theta, \sin \theta)$ propagates with wave speed $m$. Then

$$
m^{2}=\frac{B_{1}-C_{1} \cos 2(\Psi-\theta) \pm \sqrt{F(\theta)}}{2 D_{1}}
$$


where

$$
F(\theta)=\left[B_{1}-C_{1} \cos 2(\Psi-\theta)\right]^{2}-A_{1} D_{1}[\sin \phi-\cos 2(\Psi-\theta)]^{2}
$$

The two values of $m^{2}$ are real and non-negative provided that

$$
B_{1}-C_{1} \cos 2(\Psi-\theta) \geq 0 \quad \text { and } \quad F(\theta) \geq 0
$$

for all $\theta, 0 \leq \theta<\pi$. If the inequalities (56) are strict then the four roots $m$ of the characteristic equ. (53) are real and distinct and the system of pdes is hyperbolic. Let

$E_{1}=B_{1}^{2}-A_{1} D_{1} \sin ^{2} \phi, \quad F_{1}=2\left(A_{1} D_{1} \sin \phi-B_{1} C_{1}\right), \quad G_{1}=C_{1}^{2}-A_{1} D_{1}$

then

$$
\begin{gathered}
F(\theta)=G_{1} \cos ^{2} 2(\Psi-\theta)+F_{1} \cos 2(\Psi-\theta)+E_{1}, \\
=G_{1}\left(\cos 2(\Psi-\theta)+\frac{F_{1}}{2 G_{1}}\right)^{2}+\frac{4 E_{1} G_{1}-F_{1}^{2}}{4 G_{1}}
\end{gathered}
$$

provided $G_{1} \neq 0$. The following theorem is seen to hold in various cases.

Theorem 1: The minimum value $F_{\min }$ of $F(\theta)$ for $0 \leq \theta<\pi$ and the number of real roots of equ. (53), is given as follows.

(a) Let $G_{1} \neq 0$ and $\left|F_{1} / 2 G_{1}\right| \leq 1$ then the minimum value of $F(\theta)$ occurs when $\cos 2(\Psi-\theta)=-F_{1} / 2 G_{1}$ and is given by

$$
F_{\min }=\left(4 E_{1} G_{1}-F_{1}^{2}\right) / 4 G_{1}
$$

There are four distinct real roots

(i) if $G_{1}>0$ and $4 E_{1} G_{1}>F_{1}^{2}$,

(ii) if $G_{1}<0$ and $4 E_{1} G_{1}<F_{1}^{2}$.

(b) Let $G_{1} \neq 0$ and $\left|F_{1} / 2 G_{1}\right|>1$ then the minimum value of $F(\theta)$ occurs when one of $\cos 2(\Psi-\theta)= \pm 1$ holds, depending on whether $F_{1} / 2 G_{1}$ is positive or negative. Then

$$
F_{\min }=E_{1} \pm F_{1}+G_{1}
$$

depending on whether $F_{1}$ is positive or negative. There are four distinct real roots if $E_{1}+G_{1}>\mp F_{1}$.

(c) Let $G_{1}=0$ then

(i) if $F_{1}>0$ then the minimum value of $F(\theta)$ occurs at $\cos 2(\Psi-\theta)=-1$ and $F_{\text {min }}=E_{1}-F_{1}$. There are two distinct real roots if

$$
E_{1}>F_{1}, \quad B_{1} \pm C_{1}-\sqrt{E_{1}-F_{1}}>0
$$

(ii) if $F_{1}<0$ then the minimum value of $F(\theta)$ occurs at $\cos 2(\Psi-\theta)=1$ and $F_{\min }=E_{1}+F_{1}$. There are two distinct real roots if

$$
E_{1}>-F_{1}, \quad B_{1} \pm C_{1}-\sqrt{F_{1}+E_{1}}>0
$$


(iii) if $F_{1}=0$ then the minimum value is $F_{\min }=E_{1}$ and the two roots are real and distinct provided $E_{1}>0$ and if $C_{1}<0$ we have $\sqrt{E_{1}}<B_{1}+C_{1}$, while if $C_{1} \geq 0$ we have $\sqrt{E_{1}}<B_{1}-C_{1}$.

This completes the statement of the theorem.

Note that if $F_{1} \neq 0$ then in the vicinity of $G_{1}=0$ we have $\left|F_{1} / 2 G_{1}\right|>1$ and $\left|F_{1} / 2 G_{1}\right|$ is undefined at $G_{1}=0$.

The parameter space for the model is defined by the intervals

$$
0 \leq \nu \leq \frac{1}{2}, \quad 0 \leq \sin \phi<1, \quad 0 \leq \sin \nu_{p} \leq \sin \phi
$$

Although strictly speaking $\phi<\pi / 2$, we will include the limiting case $\phi=\pi / 2$ since all the coefficients are continuous in $\phi$ and this value does give some information concerning very frictional systems. Finally, it may be shown that

$$
\begin{gathered}
E_{1}=\left(1-\sin \nu_{p} \sin \phi\right)\left[\left(1-2 \nu+\sin \nu_{p} \sin \phi\right) \cos ^{2} \phi+\nu^{2}\left(1-\sin \nu_{p} \sin \phi\right)\right], \\
F_{1}=-2 \nu\left(1-\sin \nu_{p} \sin \phi\right)\left[\cos ^{2} \phi \sin \nu_{p}+\nu\left(\sin \phi-\sin \nu_{p}\right)\right] \\
G_{1}=-(1-2 \nu) \cos ^{2} \nu_{p} \cos ^{2} \phi+\nu^{2}\left(\sin \phi-\sin \nu_{p}\right)^{2} .
\end{gathered}
$$

In the above parameter space, $E_{1} \geq 0, F_{1} \leq 0$ and $G_{1}$ is of indefinite sign. Equ. (49), (50), (60) - (62) show that question of whether the model is hyperbolic is determined solely by the material parameters Poisson's ratio $\nu$, angle of internal friction $\phi$ and angle of dilatation $\nu_{p}$. It is of interest to extend the parameter space by allowing $\nu_{p}<0$ which corresponds to plastic compaction. In this case, $E_{1}, F_{1}, G_{1}$ are all of indefinite sign. However, we will not pursue this point here.

\section{Failure of Hyperbolicity for the Plastic Poten- tial Model}

Before analysing the characteristic equation for the present model in Sections 8 and 9 , we first of all demonstrate the failure of the classical plastic potential model with non-associated flow rule to exhibit hyperbolic behaviour. Let $\mu=$ 0 and also neglect the rotational terms in the Jaumann stress rate then the characteristic equ. (36) reduces to

$$
\begin{aligned}
& {\left[(\sin \phi-\cos 2 \Psi) \xi_{1}^{2}-2 \sin 2 \Psi \xi_{1} \xi_{2}+(\sin \phi+\cos 2 \Psi) \xi_{2}^{2}\right]} \\
& \left.\times\left[\left(\sin \nu_{p}-\cos 2 \Psi\right) \xi_{1}^{2}-2 \sin 2 \Psi \xi_{1} \xi_{2}+\left(\sin \nu_{p}+\cos 2 \Psi\right) \xi_{2}^{2}\right\}\right] \\
& -4\left[\left(B_{2}-C_{2} \cos 2 \Psi\right) \xi_{1}^{2}-2 C_{2} \sin 2 \Psi \xi_{1} \xi_{2}+\left(B_{2}+C_{2} \cos 2 \Psi\right) \xi_{2}^{2}\right] E^{-1}(1+\nu) \rho \bar{\xi}^{2} \\
& +4 D_{2} E^{-2}(1+\nu)^{2} \rho^{2} \bar{\xi}^{4}=0
\end{aligned}
$$

where

$B_{2}=1-\nu+\sin \phi \sin \nu_{p}, \quad C_{2}=\frac{1}{2}\left(\sin \phi+\sin \nu_{p}\right), \quad D_{2}=1-2 \nu+\sin \phi \sin \nu_{p}$, 
then in polar form,

$$
\begin{gathered}
{[\sin \phi-\cos 2(\Psi-\theta)]\left[\sin \nu_{p}-\cos 2(\Psi-\theta)\right]} \\
-4\left[\left(B_{2}-C_{2} \cos 2(\Psi-\theta)\right)\right] m^{2}+4 D_{2} m^{4}=0,
\end{gathered}
$$

and

$F(\theta)=\left[B_{2}-C_{2} \cos 2(\Psi-\theta)\right]^{2}-D_{2}[\sin \phi-\cos 2(\Psi-\theta)]\left[\sin \nu_{p}-\cos 2(\Psi-\theta)\right]$.

Now

$$
B_{2}-C_{2} \cos 2(\Psi-\theta) \geq 1-\nu+\sin \phi \sin \nu_{p}-\frac{1}{2}\left(\sin \phi+\sin \nu_{p}\right) \geq 0
$$

but

$$
[\sin \phi-\cos 2(\Psi-\theta)]\left[\sin \nu_{p}-\cos 2(\Psi-\theta)\right]<0
$$

in the four wedge-shaped regions

$$
\begin{array}{cc}
\psi+\frac{\pi}{4}+\frac{\nu_{p}}{2} \leq \theta \leq \psi+\frac{\pi}{4}+\frac{\phi}{2}, & \psi-\frac{\pi}{4}-\frac{\phi}{2} \leq \theta \leq \psi-\frac{\pi}{4}-\frac{\nu_{p}}{2} \\
\psi+\frac{5 \pi}{4}+\frac{\nu_{p}}{2} \leq \theta \leq \psi+\frac{5 \pi}{4}+\frac{\phi}{2}, & \psi+\frac{3 \pi}{4}-\frac{\phi}{2} \leq \theta \leq \psi+\frac{3 \pi}{4}-\frac{\nu_{p}}{2} .
\end{array}
$$

Hence $\sqrt{F(\theta)}>B_{2}-C_{2} \cos 2(\Psi-\theta)$ and one of the two possible values for $m^{2}$ is negative and hence two of the roots for $m$ are complex, i.e. the system is mixed. Thus, the non-associated plastic potential model predicts that, for every state of plastic stress, there are four regions arranged symmetrically about the principal axes of stress defining directions in which there are only two plane wave speeds, whereas in all other directions there are four wave speeds. This suggests a way to experimentally determine the validity or otherwise of the model. The 'blind regions' should be observable because it is reasonable to suppose that any loading which locally produces plastic deformation (e.g underground explosions or seismic events) but which far from the loading region is elastic will leave a signature in the elastic signal reflecting the existence of these regions.

\section{Hyperbolicity: Special Cases}

In the three dimensional parameter space defined by the intervals (59), taking orthogonal axes labelled $\sin \phi, \sin \nu_{p}, \nu$ the parameter space of all possible values of $\left(\sin \phi, \sin \nu_{p}, \nu\right)$ is a triangular prism with vertices at $O(0,0,0), A(1,0,0)$, $B(1,1,0), C\left(0,0, \frac{1}{2}\right), D\left(1,0, \frac{1}{2}\right)$ and $E\left(1,1, \frac{1}{2}\right)$. We shall now consider a number of important special cases of materials which correspond to edges or faces of the prism $O A B C D E$. 


\subsection{Metal plasticity}

Metal plasticity corresponds to the edge $O C$ of the parameter space prism. In this case $\phi=\nu_{p}=0,0 \leq \nu \leq \frac{1}{2}$ and neglecting the rotational terms in the Jaumann stress rate,

$$
\begin{gathered}
A_{1}=1, \quad B_{1}=1-\nu>0, \quad C_{1}=0, \quad D_{1}=1-2 \nu \geq 0 \\
E_{1}=(1-\nu)^{2}>0, \quad F_{1}=0, \quad G_{1}=-(1-2 \nu) \leq 0
\end{gathered}
$$

and so $G_{1}<0$ for $0 \leq \nu<1 / 2$ and zero at $\nu=1 / 2$. Also $B_{1}>C_{1}$.

(i) If $0 \leq \nu<1 / 2$ then $\left|F_{1} / 2 G_{1}\right|=0$ and $4 E_{1} G_{1}-F_{1}^{2}=-4(1-\nu)^{2}(1-2 \nu)<$ 0 for all values of $\nu$ in this interval. Hence the roots are real by Theorem 1(a)(ii). The wave speeds are given by

$$
m^{2}=\frac{1-\nu \pm \sqrt{(1-\nu)^{2}-(1-2 \nu) \cos ^{2} 2(\Psi-\theta)}}{2(1-2 \nu)} .
$$

(ii) if $\nu=1 / 2$ then $F_{1}=0, G_{1}=0$ and $E_{1}=1 / 4$,and there are two distinct real roots by Theorem 1 (c)(iii) given by

$$
m= \pm \frac{1}{\sqrt{2}} \cos 2(\Psi-\theta)
$$

and there is a zero wave speed in the characteristic directions

$$
\theta=\Psi \pm \frac{\pi}{4}
$$

This latter case corresponds to the classical theory of plasticity, see Hill (1950).

\subsection{Associated flow rule}

Associated flow rules correspond to the face $O B E C$, i.e. $\phi=\nu_{p}$ and neglecting the rotational terms in the Jaumann stress rate,

$$
\begin{gathered}
A_{1}=\cos ^{2} \phi, \quad B_{1}=\left(1-\nu+\sin ^{2} \phi\right) \cos ^{2} \phi \geq 0 \\
C_{1}=\cos ^{2} \phi \sin \phi \geq 0, \quad D_{1}=\cos ^{2} \phi\left(1-2 \nu+\sin ^{2} \phi\right) \geq 0 \\
E_{1}=\cos ^{4} \phi\left[(1-\nu)^{2}+\sin ^{2} \phi\right] \geq 0, \quad F_{1}=-2 \nu \cos ^{4} \phi \sin \phi \leq 0, \\
G_{1}=-(1-2 \nu) \cos ^{4} \phi \leq 0,
\end{gathered}
$$

and $G_{1}<0$ for $0 \leq \nu<1 / 2,0 \leq \phi<\pi / 2$ and $G_{1}=0$ at $\nu=1 / 2$ or $\phi=\pi / 2$.

Also, $B_{1} \geq C_{1}$.

(i) If $G_{1}<0$ then

$$
\left|\frac{F_{1}}{2 G_{1}}\right|=\frac{\nu}{1-2 \nu} \sin \phi
$$


and so may take any value $0 \leq\left|F_{1} / 2 G_{1}\right|<\infty$.

(a) If $\nu$ and $\phi$ are such that $0 \leq\left|F_{1} / 2 G_{1}\right| \leq 1$ then

$$
4 E_{1} G_{1}-F_{1}^{2}=-4 \cos ^{8} \phi(1-\nu)^{2}\left(1-2 \nu+\sin ^{2} \phi\right) \leq 0
$$

for any such $\nu$ and $\phi$ and hence the roots are real and distinct. The roots may now easily be written down using equ. (54).

(b) If $\nu$ and $\phi$ are such that $\left|F_{1} / 2 G_{1}\right|>1$ then

$$
E_{1}+G_{1}-F_{1}=\cos ^{4} \phi(\nu+\sin \phi)^{2} \geq 0
$$

and the roots are real and distinct.

(ii) If $G_{1}=0, F_{1}<0$, i.e. $\nu=1 / 2$ and $\phi<\pi / 2$, then

$$
E_{1}-F_{1}=\cos ^{4} \phi\left[\left(\frac{1}{4}+\sin \phi\right)^{2}\right] \geq 0
$$

and the roots are real and distinct.

\subsection{Plastically incompressible material}

Plastically incompressible materials correspond to the face $O A D C$ of the prism, i.e. $\nu_{p}=0$, and then

$$
\begin{gathered}
A_{1}=1, \quad B_{1}=1-\nu \geq 0, \quad C_{1}=(1-\nu) \sin \phi \geq 0, \quad D_{1}=1-2 \nu \geq 0, \\
E_{1}=(1-2 \nu) \cos ^{2} \phi+\nu^{2} \geq 0, \quad F_{1}=-2 \nu^{2} \sin \phi \leq 0, \\
G_{1}=-(1-2 \nu)+(1-\nu)^{2} \sin ^{2} \phi .
\end{gathered}
$$

$G_{1}$ is of indefinite sign and is zero for values of $\phi$ and $\nu$ which satisfy

$$
\sin ^{2} \phi=\frac{1-2 \nu}{(1-\nu)^{2}} .
$$

Also $B_{1} \geq C_{1}$. If $G_{1}<0$ then

(i) if $\left|F_{1} / 2 G_{1}\right| \leq 1$ then

$$
4 E_{1} G_{1}-F_{1}^{2}=-4(1-2 \nu)(1-\nu)^{2} \cos ^{4} \phi \leq 0
$$

is identically true and there are four real, distinct roots.

(ii) $\left|F_{1} / 2 G_{1}\right|>1$ then

$$
E_{1}+G_{1}-F_{1}=\nu^{2}(1+\sin \phi)^{2} \geq 0
$$

is identically true and there are four real, distinct roots.

If $G_{1}>0$ then $E_{1}+G_{1}-F_{1}=\nu^{2}\left(1+\sin ^{2} \phi\right) \geq 0$ holds and there are four real, distinct roots.

If $G_{1}=0, F_{1}<0$ then

$$
E_{1}+F_{1}>0
$$

and the roots are real and distinct.

Hence the model is hyperbolic for plastically incompressible granular materials. 


\subsection{Maximal limit of very frictional materials,}

The maximal limit of very frictional materials corresponds to face $A B D E, \phi=$ $\pi / 2$, and

$$
\begin{aligned}
& A_{1}=\cos ^{2} \nu_{p} \geq 0, \quad B_{1}=C_{1}=\left(1-\nu+\sin \nu_{p}\right)\left(1-\sin \nu_{p}\right) \geq 0 \\
& E_{1}=G_{1}=\nu^{2}\left(1-\sin \nu_{p}\right)^{2} \geq 0, \quad F_{1}=-2 \nu^{2}\left(1-\sin \nu_{p}\right)^{2} \leq 0 .
\end{aligned}
$$

If $G_{1}>0$ then $\left|F_{1} / 2 G_{1}\right|=1$ and

$$
4 E_{1} G_{1}-F_{1}^{2}=0
$$

and there are two real distinct roots.

If $G_{1}=0$ then $\nu_{p}=\pi / 2$ or $\nu=0$ and $E_{1}=F_{1}=0$ and there are two distinct real roots.

\subsection{Maximally elastically compressible materials,}

Maximally elastically compressible materials correspond to face $O A B, \nu=0$,

$$
\begin{gathered}
A_{1}=\cos ^{2} \nu_{p} \geq 0, \quad B_{1}=D_{1}=1-\sin ^{2} \nu_{p} \sin ^{2} \phi \geq 0, \\
C_{1}=\cos ^{2} \nu_{p} \sin \phi \geq 0 \\
E_{1}=\left(1-\sin ^{2} \nu_{p} \sin ^{2} \phi\right) \cos ^{2} \phi>0, \quad F_{1}=0, \quad G_{1}=-\cos ^{2} \nu_{p} \cos ^{2} \phi \leq 0 .
\end{gathered}
$$

Then $B_{1} \geq C_{1}$.

(i) If $G_{1}<0$ then $\left|F_{1} / 2 G_{1}\right|=0$ so $4 E_{1} G_{1} \leq F_{1}^{2}$ is identically satisfied giving four real, distinct roots.

(ii) If $G_{1}=0$ (the limiting cases $\nu_{p}=\pi / 2$ or $\phi=\pi / 2$ ) then $0 \leq\left|F_{1} / 2 G_{1}\right|<\infty$. If $\left|F_{1} / 2 G_{1}\right| \leq 1$ then $4 E_{1} G_{1}-F_{1}^{2}=0$ and there are two real distinct roots.

If $\left|F_{1} / 2 G_{1}\right|>1$ then $E_{1}+G_{1}-F_{1}=\left(1-\sin ^{2} \nu_{p} \sin ^{2} \phi\right) \cos ^{2} \phi \geq 0$ and there are two real distinct roots.

\subsection{Elastically incompressible materials,}

Elastically incompressible materials correspond to face $C D E, \nu=1 / 2$,

$$
\begin{gathered}
A_{1}=\cos ^{2} \nu_{p} \geq 0, \quad B_{1}=\left(\frac{1}{2}+\sin \nu_{p} \sin \phi\right)\left(1-\sin \nu_{p} \sin \phi\right) \geq 0 \\
C_{1}=\cos ^{2} \nu_{p} \sin \phi-\frac{1}{2}\left(\sin \phi-\sin \nu_{p}\right), \\
D_{1}=\left(1-\sin \nu_{p} \sin \phi\right) \sin \nu_{p} \sin \phi \geq 0 \\
E_{1}=\frac{1}{4}\left(1-\sin \nu_{p} \sin \phi\right)\left[1+\sin \nu_{p} \sin \phi(1+2 \cos 2 \phi)\right] \geq 0, \\
F_{1}=-\frac{1}{2}\left(1-\sin \nu_{p} \sin \phi\right)\left(\sin \phi+\cos 2 \phi \sin \nu_{p}\right) \leq 0,
\end{gathered}
$$




$$
G_{1}=\frac{1}{4}\left(\sin \phi-\sin \nu_{p}\right)^{2} \geq 0 .
$$

Also $B_{1} \pm C_{1} \geq 0$. If $G_{1}>0$ then $\left|F_{1} / 2 G_{1}\right| \geq 1$ and $E_{1}+G_{1}-F_{1} \geq 0$. There are four real, distinct roots.

If $G_{1}=0$ then $\phi=\nu_{p}$. If $F_{1}<0$ then

$$
\begin{gathered}
E_{1}=\frac{1}{4} \cos ^{2} \phi\left(1+4 \sin ^{2} \phi\right) \geq 0, \\
F_{1}=-\cos ^{4} \phi \sin \phi \leq 0, \\
E_{1}-F_{1}=\cos ^{4} \phi\left(\frac{1}{2}-\sin \phi\right)^{2} \geq 0
\end{gathered}
$$

and there are 4 distinct real roots.

If $F_{1}=0$ then $\phi=0$ or $\phi=\pi / 2$ and there are 2 distinct real roots.

\section{Hyperbolicity: the General Case}

Having considered the above special cases, we now consider the remaining case and consider the interior of the prism $O A B C D E$ using equ. (48) - (51), (60) (62).

Note that $B_{1} \pm C_{1} \geq 0 . G_{1}$ is of indefinite sign and is zero for values of $\phi, \nu_{p}$ and $\nu$ which satisfy

$$
\frac{\nu^{2}}{1-2 \nu}=\frac{\cos ^{2} \nu_{p} \cos ^{2} \phi}{\left(\sin \phi-\sin \nu_{p}\right)^{2}} .
$$

Case (a) If $\left|F_{1} / 2 G_{1}\right|>1$ then (irrespective of the sign of $G_{1}$ )

$$
\begin{aligned}
E+G-F= & \cos ^{4} \phi \sin ^{2} \nu_{p}+2 \nu \cos ^{2} \phi(1+\sin \phi)\left(1-\sin \nu_{p}\right) \sin \nu_{p} \\
& +\nu^{2}\left(1-\sin \nu_{p} \sin \phi+\sin \phi-\sin \nu_{p}\right)^{2} \geq 0
\end{aligned}
$$

and the inequality is automatically satisfied.

Case (b) If $\left|F_{1} / 2 G_{1}\right| \leq 1$ then

(i) If $G_{1}<0$ then $4 E_{1} G_{1} \leq F_{1}^{2}$ is automatically satisfied.

(ii) There are no values of $\nu, \phi, \nu_{p}$ satisfying $G_{1}>0$.

Hence, we conclude that the model is hyperbolic over the whole of the parameter space. Given that the model is constructed from two models which fail to be hyperbolic, together with a general tendency for models of granular materials to exhibit ill-posedness of one sort or another, this is a striking result.

\section{Equations Governing the Cosserat Model}

The model described in Section 3, with $\boldsymbol{\Omega}$ taken to be identically zero relative to a suitable frame, is sufficient for many applications. For planar flows in which both $\dot{\psi} \equiv 0$ and $\boldsymbol{\Omega}=\mathbf{0}$ the model is identical to the double-shearing model. 
In this Section we complete the model of Section 3 by introducing equations which govern the intrinsic spin $\boldsymbol{\Omega}$. The proper context for this quantity is a Cosserat continuum and a complete Cosserat model is needed to investigate the internal structure of shear bands and also in the neighbourhood of rigid or elastic external boundaries. A model for classical flows is then obtained by putting $\boldsymbol{\Omega}=\mathbf{0}$ relative to a suitable frame $F_{1}$. For a frame $F_{2}$ rotating steadily relative to $F_{1}, \boldsymbol{\Omega}$ takes a non-zero constant value determined by the relative spin of the two frames. In this section we embed the model for classical flows into a special Cosserat model which preserves the characteristic structure. The couple stress tensor is denoted by $\boldsymbol{\mu}=\left(\mu_{i j}\right)$, the angular momentum by $\mathbf{l}$, where $\mathbf{l}=\mathbf{I} \cdot \boldsymbol{\Omega}$, and $\mathbf{I}$ denotes the moment of inertia density tensor. In a classical continuum, the Cauchy stress $\boldsymbol{\sigma}$ is symmetric but will, in general, be non-symmetric in a Cosserat continuum. Let $\boldsymbol{\sigma}^{s}, \boldsymbol{\sigma}^{a}$ denote the symmetric and anti-symmetric parts of the Cauchy stress. In a classical continuum $\boldsymbol{\sigma}$ and $\boldsymbol{\sigma}^{s}$ coincide and $\boldsymbol{\sigma}^{a}=\mathbf{0}$.

The equations of Section 3 labeled (a) and (b) still hold, while (c) (d) become as follows.

$\left(\mathrm{c}^{\prime}\right)$ The yield condition $(\mathrm{c})$ becomes

$$
f(\boldsymbol{\sigma}, \boldsymbol{\mu}) \leq 0,
$$

where $f$ denotes a scalar valued function of the stress $\boldsymbol{\sigma}$ and couple stress $\boldsymbol{\mu}$. At a point $P$, if $f(\boldsymbol{\sigma}, \boldsymbol{\mu})<0$, the material is in an elastic state, while if $f(\boldsymbol{\sigma}, \boldsymbol{\mu})=0$ the material is in a state of yield (or in a plastic state). Define

$$
\mathbf{f}^{\sigma}=\left(f_{i j}^{\sigma}\right)=\frac{\partial f^{\sigma}}{\partial \boldsymbol{\mu}}=\left(\frac{\partial f^{\sigma}}{\partial \mu_{i j}}\right), \quad \mathbf{f}^{\mu}=\left(f_{i j}^{\mu}\right)=\frac{\partial f^{\mu}}{\partial \boldsymbol{\mu}}=\left(\frac{\partial f^{\mu}}{\partial \mu_{i j}}\right),
$$

then the condition for continuing yield is

$$
\mathbf{f}^{\sigma} \cdot \stackrel{\circ}{\boldsymbol{\sigma}}+\mathbf{f}^{\mu} \cdot \stackrel{\leftrightarrow}{\boldsymbol{\mu}}=0, \quad f_{i j}^{\sigma} \stackrel{\circ}{\sigma}_{i j}+f_{i j}^{\mu} \stackrel{\circ}{\mu}_{i j}=0 .
$$

$\left(\mathrm{d}^{\prime}\right)$ The flow rule (d) still holds (we will now refer to it as the translational flow rule) with $\mathbf{g}=\mathbf{g}^{\sigma}(\boldsymbol{\sigma}, \boldsymbol{\mu})$ defined analogously to $\mathbf{f}^{\sigma}$.

Now consider the equations governing the rotational part of the motion.

(e) Euler's equations of rotational motion are

$$
\nabla \cdot \boldsymbol{\mu}+\rho \mathbf{G}+\boldsymbol{\varepsilon}: \boldsymbol{\sigma}=\rho d_{t} \mathbf{l}, \quad \partial_{j} \mu_{j i}+\rho G_{i}+\epsilon_{i j k} \sigma_{j k}=\rho d_{t} l_{i}
$$

where $\mathbf{G}$ denotes the body couple.

(f) For the couple stress flow rule, let

$$
\mathbf{e}=\nabla \boldsymbol{\Omega}^{v}
$$

recalling that $\boldsymbol{\Omega}^{v}=\left(\Omega_{i}\right)$, denotes the vector form of the intrinsic spin. The intrinsic spin gradient $\mathbf{e}$ is frame indifferent. The rotational flow rule is taken to be

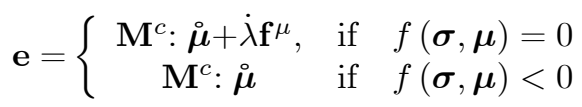


where $\mathbf{M}^{c}$ denotes the 4 th order elastic compliance tensor, $\stackrel{\boldsymbol{\mu}}{\text { the Jaumann }}$ derivative of the couple stress.

We state here an explicit, simple model based on the following special assmptions:

(i) Granular materials are not capable of sustaining a non-symmetric Cauchy stress, $\boldsymbol{\sigma}^{a}=\mathbf{0}$

(ii) The couple stress tensor is anti-symmetric;

(iii) The constitutive equations may be decomposed into translational and rotational parts. Thus, we shall assume separate translational and rotational yield conditions

$$
f^{s}(\boldsymbol{\sigma}) \leq 0, \quad f^{a}(\boldsymbol{\mu}) \leq 0 .
$$

A full discussion of the possibilities of embedding the classical model into a heirarchy of Cosserat models will be presented in a subsequent paper. For a planar deformation in the $O x_{1} x_{2}$ plane the equations are as follows. The spin equations of motion reduce to the single equation

$$
\partial_{1} \mu_{13}+\partial_{2} \mu_{23}+\rho G_{3}=\rho d_{t} l_{3}
$$

since all quantities are independent of $x_{3}$. In this model $\sigma_{12}=\sigma_{21}$. The equation of continuing rotational yield reads

$$
f_{13}^{a} \stackrel{\leftrightarrow}{\mu}_{13}+f_{23}^{a} \stackrel{\leftrightarrow}{\mu}_{23}=0 .
$$

The couple stress flow rule is

$$
\begin{aligned}
& \partial_{1} \Omega_{3}=M_{13}^{c} \stackrel{\circ}{\mu}_{13}+\dot{\lambda}^{c} f_{13}^{a}, \\
& \partial_{2} \Omega_{3}=M^{c} \stackrel{\mu}{\mu}_{23}+\dot{\lambda}^{c} f_{23}^{a} .
\end{aligned}
$$

where $M^{c}$ denotes the couple stress elastic modulus, $\dot{\lambda}^{c}$ a non-negative multiplier. The unknown quantities are: $v_{1}, v_{2}, \sigma_{11}, \sigma_{12}, \sigma_{22}, \rho, \mu_{13}, \mu_{23}, \Omega_{3}, \dot{\lambda}, \dot{\lambda}^{c}$. The equations comprise 2 translational equations of motion, 1 rotational equation of motion, 3 translational constitutive equations, 2 rotational constitutive equations, 1 continuity continuity equation and 2 continuing yield equations. A variant involving a single continuing yield equation and $\dot{\lambda}=\dot{\lambda}^{c}$ will be needed if it turns out that experimental properties of granular materials require coupling between the translational and rotational variables to describe the interior structure of a shear band.

\section{Linearisation of the Cosserat Model Equa- tions}

Now define

$$
\mathbf{z}^{T}=\left(v_{1}, v_{2}, \dot{\lambda}, \sigma_{11}, \sigma_{12}, \sigma_{22}, \rho, \Omega_{3}, \dot{\lambda}^{c}, \mu_{13}, \mu_{23}\right)
$$

and let

$$
\mathbf{Z}^{T}=\left(V_{1}, V_{2}, \dot{\Lambda}, \Sigma_{11}, \Sigma_{12}, \Sigma_{22}, \varrho, \Omega_{3}^{u}, \dot{\Lambda}^{c}, M_{13}, M_{23}\right)
$$


denote a given solution of equations (10), (12), (13), (16) - (18), (71) - (74). Let

$$
\mathbf{z}^{\prime T}=\left(v_{1}^{\prime}, v_{2}^{\prime}, \dot{\lambda}^{\prime}, \sigma_{11}^{\prime}, \sigma_{12}^{\prime}, \sigma_{22}^{\prime}, \rho^{\prime}, \Omega_{3}^{\prime}, \dot{\lambda}^{c \prime}, \mu_{13}^{\prime}, \mu_{23}^{\prime}\right)
$$

denote an arbitrary perturbation of the given solution. Then define

$$
\Omega_{3}=\Omega_{3}^{u}+\Omega_{3}^{\prime}, \quad \dot{\lambda}^{c}=\dot{\Lambda}^{c}+\dot{\lambda}^{c \prime}, \quad \mu_{13}=M_{13}+\mu_{13}^{\prime}, \quad \mu_{23}=M_{23}+\mu_{23}^{\prime} .
$$

Linearising equations (10), (12), (13), (16) - (18), (71) - (74)) and using the fact that $\mathbf{Z}$ is a solution gives the following system of linear pdes for $\mathbf{z}^{\prime}$. Denote coefficients of components $\mathbf{z}^{\prime}$ evaluated in the given solution $\mathbf{Z}$ which are of zeroth order in derivatives of components of $\mathbf{z}^{\prime}$, by $c_{i j}$, where $i$ denotes the equation and $j$ the component $\mathbf{z}^{\prime}$. There is now an extra term involving $\Omega_{3}^{\prime}$ in the linearisation of the translational flow rule of Section 3.

(a) First component of the translational flow rule

$$
\begin{gathered}
\left(-\partial_{1}+\Delta_{1} \partial_{2}+c_{41}\right) v_{1}^{\prime}+\left(-\Delta_{1} \partial_{1}+c_{42}\right) v_{2}^{\prime}+G_{11} \dot{\lambda}^{\prime} \\
+\left(a D_{t}+c_{44}\right) \sigma_{11}^{\prime}+c_{45} \sigma_{12}^{\prime}+\left(b D_{t}+c_{46}\right) \sigma_{22}^{\prime}+c_{48} \Omega_{3}^{\prime}=0,
\end{gathered}
$$

where

$$
c_{48}=2 \mu \Sigma_{12} .
$$

(b) Second component of the translational flow rule

$$
\begin{gathered}
{\left[-\left(1+\Delta_{2}\right) \partial_{2}+c_{51}\right] v_{1}^{\prime}+\left[\left(-1+\Delta_{2}\right) \partial_{1}+c_{52}\right] v_{2}^{\prime}+2 G_{12} \dot{\lambda}^{\prime}} \\
+c_{54} \sigma_{11}^{\prime}+\left(c D_{t}+c_{55}\right) \sigma_{12}^{\prime}+c_{56} \sigma_{22}^{\prime}+c_{58} \Omega_{3}^{\prime}=0
\end{gathered}
$$

where

$$
c_{58}=-2 \mu\left(\Sigma_{11}-\Sigma_{22}\right) .
$$

(c) Third component of the translational flow rule

$$
\begin{gathered}
\left(-\Delta_{1} \partial_{2}+c_{61}\right) v_{1}^{\prime}+\left(-\partial_{2}+\Delta_{1} \partial_{1}+c_{62}\right) v_{2}^{\prime}+G_{22} \dot{\lambda}^{\prime} \\
+\left(b D_{t}+c_{64}\right) \sigma_{11}^{\prime}+c_{65} \sigma_{12}^{\prime}+\left(a D_{t}+c_{66}\right) \sigma_{22}^{\prime}+c_{68} \Omega_{3}^{\prime}=0,
\end{gathered}
$$

where

$$
c_{68}=-2 \mu \Sigma_{12} .
$$

(d) Equation of rotational motion

$$
c_{81} v_{1}^{\prime}+c_{82} v_{2}^{\prime}+c_{87} \rho^{\prime}+I \varrho D_{t} \Omega_{3}^{\prime}-\partial_{1} \mu_{13}^{\prime}-\partial_{2} \mu_{23}^{\prime}=0,
$$

where

$$
c_{81}=I \varrho \partial_{1} \Omega_{3}, \quad c_{82}=I \varrho \partial_{2} \Omega_{3}, \quad c_{87}=I D_{t} \Omega_{3}-G_{3} .
$$

(e) Rotational continuing yield

$$
\left(F_{13}^{a} D_{t}+c_{9,11}\right) \mu_{13}^{\prime}+\left(F_{23}^{a} D_{t}+c_{9,12}\right) \mu_{23}^{\prime}=0,
$$


where

$$
\begin{aligned}
c_{9,11} & =\left.\frac{\partial f_{13}^{a}}{\partial \mu_{13}}\right|_{\mathbf{M}} D_{t} M_{13}+\left.\frac{\partial f_{23}^{a}}{\partial \mu_{13}}\right|_{\mathbf{M}} D_{t} M_{23}, \\
c_{9,12} & =\left.\frac{\partial f_{13}^{a}}{\partial \mu_{23}}\right|_{\mathbf{M}} D_{t} M_{13}+\left.\frac{\partial f_{23}^{a}}{\partial \mu_{23}}\right|_{\mathbf{M}} D_{t} M_{23} .
\end{aligned}
$$

(f) First component of couple stress flow rule

$$
-\partial_{1} \Omega_{3}^{\prime}+F_{13}^{a} \dot{\lambda}^{c \prime}+\left(M^{c} D_{t}+c_{10,10}\right) \mu_{13}^{\prime}+c_{10,11} \mu_{23}^{\prime}=0,
$$

where

$$
c_{10,10}=\left.\dot{\Lambda}^{c} \frac{\partial f_{13}^{a}}{\partial \mu_{13}}\right|_{\mathbf{M}}, \quad c_{10,11}=\left.\dot{\Lambda}^{c} \frac{\partial f_{13}^{a}}{\partial \mu_{23}}\right|_{\mathbf{M}} .
$$

(g) Second component of couple stress flow rule

$$
-\partial_{2} \Omega_{3}^{\prime}+F_{23}^{a} \dot{\lambda}^{c \prime}+c_{11,10} \mu_{13}^{\prime}+\left(M^{c} D_{t}+c_{11,11}\right) \mu_{23}^{\prime}=0,
$$

where

$$
c_{11,10}=\left.\dot{\Lambda}^{c} \frac{\partial f_{23}^{a}}{\partial \mu_{13}}\right|_{\mathbf{M}} \quad c_{11,11}=\left.\dot{\Lambda}^{c} \frac{\partial f_{23}^{a}}{\partial \mu_{23}}\right|_{\mathbf{M}} .
$$

Writing the linearised Cosserat equations in block matrix form gives

$$
\left[\begin{array}{ll}
A & D \\
C & B
\end{array}\right] \mathbf{z}^{\prime}=\mathbf{0}
$$

where

$$
\begin{aligned}
& B=\left[\begin{array}{cccc}
I \varrho D_{t} & 0 & -\partial_{1} & -\partial_{2} \\
0 & 0 & F_{13}^{a} D_{t}+c_{9,11} & F_{23}^{a} D_{t}+c_{9,12} \\
-\partial_{1} & F_{13}^{a} & M^{c} D_{t}+c_{10,10} & c_{10,11} \\
-\partial_{2} & F_{23}^{a} & c_{11,10} & M^{c} D_{t}+c_{11,11}
\end{array}\right] \\
& C=\left[\begin{array}{ccccccc}
c_{81} & c_{82} & 0 & 0 & 0 & 0 & c_{87} \\
0 & 0 & 0 & 0 & 0 & 0 & 0 \\
0 & 0 & 0 & 0 & 0 & 0 & 0 \\
0 & 0 & 0 & 0 & 0 & 0 & 0
\end{array}\right] \\
& D=\left[\begin{array}{cccc}
0 & 0 & 0 & 0 \\
0 & 0 & 0 & 0 \\
0 & 0 & 0 & 0 \\
c_{48} & 0 & 0 & 0 \\
c_{58} & 0 & 0 & 0 \\
c_{68} & 0 & 0 & 0 \\
0 & 0 & 0 & 0
\end{array}\right]
\end{aligned}
$$

and $B$ has symbol

$$
B=\left[\begin{array}{cccc}
I \varrho i \bar{\xi} & 0 & -i \xi_{1} & -i \xi_{2} \\
0 & 0 & F_{13}^{a} i \bar{\xi}+c_{9,11} & F_{23}^{a} i \bar{\xi}+c_{9,12} \\
-i \xi_{1} & F_{13}^{a} & M^{c} i \bar{\xi}+c_{10,10} & c_{10,11} \\
-i \xi_{2} & F_{23}^{a} & c_{11,10} & M^{c} i \bar{\xi}+c_{11,11}
\end{array}\right]
$$

with principal part $B^{p}$ obtained by replacing all $c_{i j}$ by zero. 


\section{Hyperbolicity of the Cosserat Model}

The principal part of the symbol obtained by expanding the determinant of the matrix in eq. (82) gives

$$
\operatorname{det} A^{p}=0, \quad \operatorname{det} B^{p}=0
$$

i.e. the characteristic equation for classical flows together with the characteristic equation for the Cosserat equations. Expanding the latter gives either $\dot{\xi}=0$ or

$$
M^{c} I \varrho\left[\left(F_{13}^{a}\right)^{2}+\left(F_{23}^{a}\right)^{2}\right] \bar{\xi}^{2}-\left(\xi_{1} F_{23}^{a}-\xi_{2} F_{13}^{a}\right)^{2}=0
$$

which has two distinct real roots. This establishes that the model for classical flows can be embedded in a simple Cosserat model which is hyperbolic and in which the characteristic structure of the classical model is preserved. This gives a base model from which more complicated Cosserat models can be constructed to obtain agreement with any experimental evidence that becomes available.

\section{Conclusions}

It has been established that a model for the deformation and flow of granular materials comprising the plastic potential model augmented with a threedimensional analogue of the "non-coaxial" term of the double-shearing model in which the planar stress-rate $d_{t} \psi$ is replaced by the vector (in three dimensions) intrinsic spin $\Omega$ is hyperbolic, i.e. the roots of the characteristic equation are real and distinct. The Cauchy problem for the model is hence well-posed. The formulation of the model governing classical flows requires a Cosserat quantity to take degenerate values and the model has been embedded into a simple model for a Cosserat continuum. The Cosserat model has been constructed in such a way that the underlying characteristic structure for classical flows is preserved.

It is emphasised that the model is constructed on the basis of two models which are well-established in describing observed and experimental behaviour of granular materials. The new model has the considerable advantage of being well-posed for the Cauchy problem, whereas the two previous models are both ill-posed. As examples of applications of its use, the new model is capable of analysing elasto-plastic wave propagation in soils and granular materials and may also be used for quasi-static rigid-plastic flows. Again, neither of the models from which it was derived are capable of doing this.

The major conclusion of the work presented in this paper is that the first interpretation of the quote by A.J.M. Spencer given in the introduction is now no longer true: a robust model suitable for the analysis of a wide range of problems in the mechanics of granular materials and soil mechanics is now available to researchers. 


\section{Bibliography}

Anand, L. 1983 Plane deformations of ideal granular materials, J Mech Phys Solids 31, 105-122.

de Josselin de Jong, G. 1959 Statics and Kinematics of the failable zone of a granular material, Delft: Uitgeverij Waltmann.

de Josselin de Jong, G. 1977 Mathematical elaboration of the double-sliding, free rotating model, Arch. Mech. 29, 561-591.

Drucker, D.C. 1951, A more fundamental approach to plastic stress-strain relations. In Proc. 1st. U.S. Natl. Congr. Appl. Mech., (ed. E. Sternberg) pp. 487-491. ASME: New York.

Drucker, D.C. \& Prager, W. 1952 Soil mechanics and plastic analysis or limit design, Q Appl Math 10, 157-165.

Geniev, G.A., 1958 Problems of the dynamics of a granular mediun (in Russian), Akad Stroit Archit SSSR, 3-121 Moscow.

Harris, D 1993 Constitutive equations for planar deformations of rigid-plastic materials, J Mech Phys Solids 41, 1515-1531.

Harris, D. 1995 A unified formulation for plasticity models of granular and other materials, Proc Roy Soc Lond A 450, 37-49.

Harris, D. 2001 Ill- and well-posed models of granular flow, Acta Mechanica 146, 199-225.

Harris, D. 2001 Characteristic relations for a model for the flow of granular materials, Proc R Soc Lond A 457, 349-370.

Harris, D. \& Grekova, E.F. 2005 A hyperbolic well-posed model for the flow of granular materials, Jnl Engineering Mathematics 52, 107-135.

Harris, D. 2006 Double-slip and spin: dilatant shear in a reduced Cosserat model. In Modern trends in geomechanics (ed. W. Wu and H.S.Yu), pp. 329346 Berlin: Springer.

Hill, R. 1950 The mathematical theory of plasticity, Oxford: Clarendon Press. Ilyushin, A.A. 1961 On the postulate of plasticity (in Russian), Prikl. Math. Mekh., 25, 503-507.

Kruyt, N.P. 1990 An analysis of the generalized double-sliding models for cohesionless granular materials, J Mech Phys Solids 38, 27-35.

Mandel, J. 1947 Sur les lignes de glissement et le calcul des déplacements dans la déformation plastique, C.R. Acad Sci Paris 225, 1272-1273.

Mehrabadi, M.M. \& Cowin, S.C. 1978 Initial planar deformation of dilatant granular materials, J Mech Phys Solids 26, 269-284.

Mroz, Z., \& Szymansk, C. 1979 Non-associated flow rules in description of plastic flow of granular materials, CISM Course 217 (1974). In Limit analysis and rheological approach in soil mechanics (ed. W. Olszak and L. Sukjle), Vienna: Springer.

Pitman, E.B. \& Schaeffer, D.G. 1987 Stability of time dependent compressible 
granular flow in two dimensions, Comm Pure Appl Math 40, 421-447.

Schaeffer, D.G. \& Pitman, E.B. 1988 Ill-posedness in three dimensional plastic flow, Comm Pure Appl Math 41, 879-890.

Schaeffer, D.G. 1990 Mathematical issues in the continuum formulation of slow granular flow. In Two phase waves in fluidised beds, sedimentation and granular flows (ed. D.D. Joseph \& D.G. Schaeffer), pp. 118-129. Minneapolis: Institute of Mathematics and its Applications, University of Minnesota.

Spencer, A.J.M. 1964 A theory of the kinematics of ideal soils under plane stran conditions, J Mech Phys Solids 12, 337-351.

Spencer, A.J.M. 1982 Deformation of ideal granular materials. In The Rodney Hill 60th. anniversary volume, (ed. H.G. Hopkins and M.J. Sewell), pp. 607-652. Oxford: Pergamon. 